\title{
Routine use of ultrasound guided tandem placement in intracavitary brachytherapy for the treatment of cervical cancer - a South Indian institutional experience
}

\author{
Pamidimukkala Bramhananda Rao, MD, Assoc. Prof., Saptarshi Ghosh, MD \\ Department of Radiotherapy, GSL Cancer Hospital, GSL Medical College, Rajahmundry. Andhra Pradesh, India
}

\begin{abstract}
Purpose: Intracavitary brachytherapy necessitates the insertion of a tandem applicator through the cervical os into the uterine cavity. Blind insertion of the tandem may result in suboptimal tandem placement. This decreases the control of the tumor locally and may result in uterine perforation. Although routine real time ultrasound guided tandem placement has shown better results, it is seldom practised. The aim of this work is to evaluate the role of routine real-time intraoperative trans-abdominal ultrasound guided tandem placement in intracavitary brachytherapy for the treatment of cervical cancer.

Material and methods: This is a prospective single institutional study conducted from April 2013 to May 2015. A total of 96 patients of locally advanced cervical cancer were treated with routine ultrasound guided brachytherapy amounting to a total of 282 intracavitary applications.

Results: In 78 of the study patients, the cervical os could be easily identified visually, which was then confirmed with ultrasound guidance. In another 12 patients, though the os could be identified visually, uterine sounding was only possible under ultrasound guidance. In another 4 patients, the cervical os could not be identified visually as the cervix was flushed with vagina and ultrasound guidance was necessary for accurate os identification. In 2 of the study patients, intraoperative ultrasound helped in identifying the patients suitable for interstitial brachytherapy rather than intracavitary brachytherapy. Out of the 96 study patients, the length of the uterine canal changed in 15 patients during the subsequent brachytherapy application.

Conclusions: This procedure is strongly advocated for proper placement of the tandem applicator and to avoid perforations. It is an accurate, fast, easily available, and cost-effective method. Hence, it can be incorporated in intracavitary applications for cervical cancers even in the developing countries where cost, accessibility, and time are important issues.
\end{abstract}

Key words: brachytherapy, cervical cancer, tandem, ultrasound guidance.

\section{Purpose}

Cervical cancer is the second most common malignancy in India [1]. Locally advanced cervical cancers are treated with external beam radiation therapy (EBRT) along with cisplatin based concurrent chemotherapy and brachytherapy [2, 3]. Brachytherapy forms an integral part in the treatment of cervical cancers [4]. It boosts the tumor, while sparing the surrounding organs as much as possible. Recently, the role of brachytherapy boost following complete treatment of cervical cancer, based on the presence of residual malignant cells in biopsy has also been addressed [5]. The International Commission on Radiation Units and Measurement (ICRU) 38 Recommendations have attempted using radiographs for treatment planning and uniform reporting of intracavitary brachytherapy (ICBT) [6]. In ICBT, a central tandem applicator is gently inserted through the cervical os into the uterine cavity. In most instances, this is done blindly by carefully passing the tandem until a slight sense of resistance is felt by the brachytherapist. This sense of resistance implies that the tip of the tandem has reached the uterine fundus $[7,8,9]$. Improper placement of the central tandem applicator results in poor local tumor control and uterine perforations. It may also result in higher radiation doses to the organs at risk [10]. Many risk factors
Address for correspondence: Saptarshi Ghosh, MD, Department of Radiotherapy, GSL Trust Cancer Hospital and Research Centre, Near Kambala Park, 533105 Rajahmundry, India, phone: +91 8106572241 , ๑-mail: drsaptarshi10@gmail.com
Received: 14.07.2015

Accepted: 25.09.2015

Published: 31.10 .2015 
have been identified for improper tandem placement: 1) patients aged 60 and above, 2) history of prior cervical surgery, 3) cervical os stenosis, 4) cervical anatomical distortion, 5) retroversion of the uterus, and 6) increased disease extent $[7,11,12,13,14,15,16]$. The risk of uterine perforation with the blind insertion procedure ranges from $2-14 \%$, according to different studies [7, 11, 12, $13,16]$. One technique of verifying real-time tandem insertion is intraoperative ultrasonography $[12,14]$. Tansabdominal ultrasound is used to determine the size, shape, thickness, and diameter of uterus, cervix, and cervical cancer along with the parametrial involvement [17]. Intraoperative ultrasound guided tandem placement decreases the risk of uterine perforation and improves local control of the tumor $[7,18,19]$. Although this procedure has its benefits, an American survey shows that it has been practiced in only $42 \%$ of ICBT applications [20] The procedure is rarely applied in developing countries.

The objective of this study is to evaluate the role of this procedure in terms of identification of os, determination of the length of the uterine canal, and decision to change the application to interstitial brachytherapy (thereby reducing the risk of perforations).

\section{Material and methods}

The current study is a prospective single institutional study carried out from April 2013 to May 2015. A total of 282 intracavitary applications were performed under ultrasound guidance in 96 patients of locally advanced cervical cancer. The patients had locally advanced cervical cancer (FIGO Stages $\mathrm{IB}_{2}$ - IIIB), and underwent real-time ultrasound guided tandem applicator placement. All the applications were performed by the same brachytherapist (the first author in the present study). The study patients were treated with EBRT up to a dose of 50.4 Gy at 1.8 Gy per fraction in 28 fractions with 5 fractions per week in parallel opposed antero-posterior and postero-anterior fields. Midline shielding with $4 \mathrm{~cm}$ central shield was done after 25 fractions in all patients, as cumulative dose differs with difference in size of the central shielding [21]. Concurrent chemotherapy was delivered with weekly Cisplatin at $40 \mathrm{mg} / \mathrm{m}^{2}$. This was followed by a boost of 7 Gy per weekly fraction of high dose-rate (HDR) ICBT in three applications. The total dose in 2 Gy per fraction equivalent (EQD2) to Point A was 74 Gy [22], according to the institutional protocol.

\section{Technique}

All patients underwent bimanual pelvic examination under mild intravenous anesthesia for the evaluation of residual primary tumor and cervical os identification. The os was first tried to identify by uterine sounding with naked eye. This was followed by ultrasound examination to confirm the os position. Foley's catherisation was performed to drain out as much of urine by gently pressing the abdomen. For better visualization of the bladder, $200-300 \mathrm{ml}$ of normal saline was instilled into the urinary bladder retrogradely. The catheter was clamped with the saline filling the urinary bladder. Real-time intraoperative transabdom- inal ultrasound scanning was done with Philips HD 7 machine (Philips Healthcare, Amsterdam, The Netherlands) with curvilinear $5 \mathrm{MHz}$ probe. The cervical os was then sounded under ultrasound guidance. After cervical os dilatation, the tandem was gently inserted through the os into the uterine cavity (Figure 1). The angulation and length of the tandem were determined from the extent of uterine flexion and the distance of the fundus from the cervical os on ultrasound scanning respectively.

The bladder was then drained. Vaginal colpostats of maximum possible ovoid diameter were inserted gently avoiding vaginal lacerations as much as possible. The geometry of the tandem relative to the vaginal colpostats was confirmed visually before fixing the device. Intraoperative ultrasound was discontinued after confirming the final tandem position. This was followed by vaginal packing with radiopaque gauze in a proximal-to-distal fashion, displacing the bladder anteriorly and rectum posteriorly as much as possible, without disturbing the applicator geometry. Postoperative ultrasound scanning and clinical examination were performed in all patients to rule out any uterine perforation. In doubtful cases, CT scans were performed. Although image based brachytherapy is now quite a norm in the developed countries [23], orthogonal radiographs were used in our institution for treatment planning due to cost implications. A western study has reported larger dosimetric impact of tandem-and-ovoid displacements in MRI-guided conformal brachytherapy plans than in the conventional Point A plans [24]. All the current study patients were treated on HDR MicroSelectron (Nucletron, an Elekta company, Elekta AB, Stockholm, Sweden) brachytherapy machine with Oncentra planning system (Nucletron).

\section{Results}

Figure 2 depicts the role of ultrasound guided tandem insertion in the study patients. Ninety-six patients underwent routine ultrasound guided tandem placement in the present study. The cervical os was accurately identified in 91 patients visually, which was then confirmed with ultrasound guidance. In 78 of these patients, the uterine sound was inserted without any ultrasound guidance into the uterine canal accurately. The position of the uterine sound in the uterine canal was confirmed with ultra-

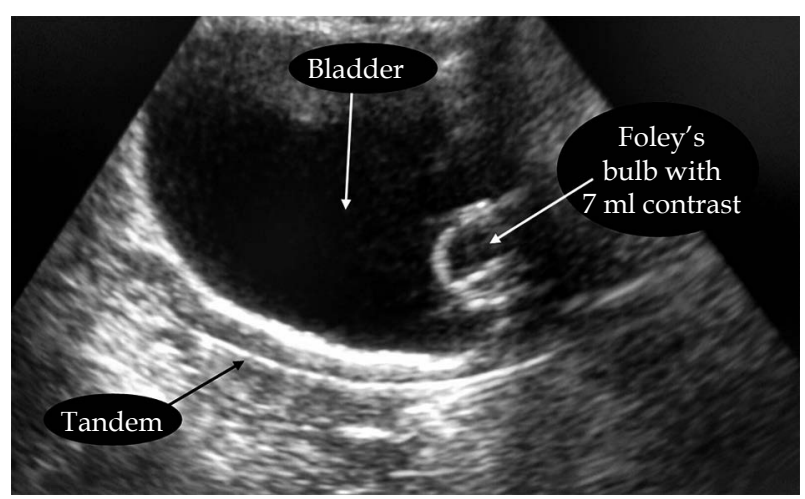

Fig. 1. Image of tandem placed in accurate position as visualized on transabdominal ultrasound 


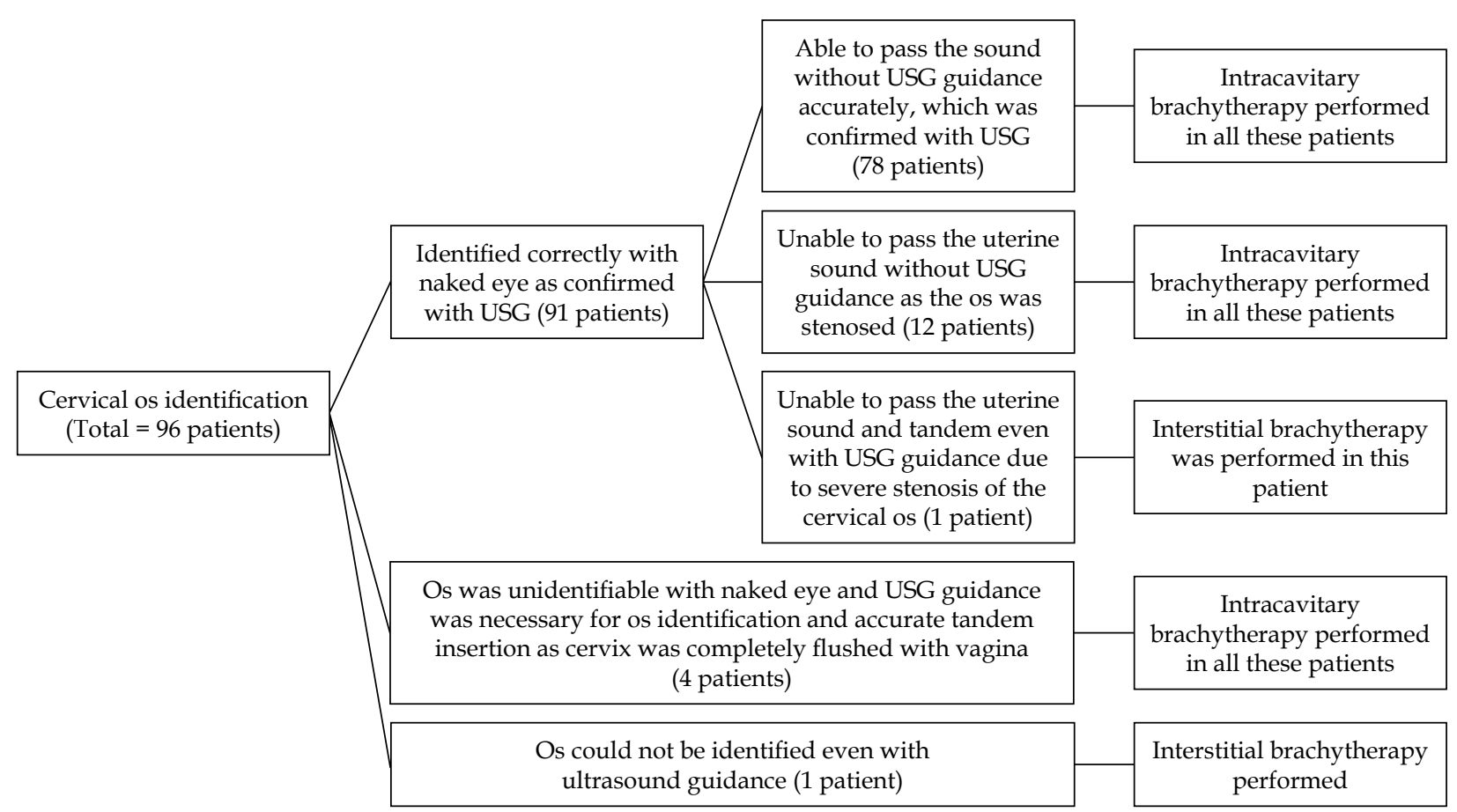

Fig. 2. Tandem insertion and the role of ultrasound guidance in the study patients

sound. In these patients, the benefit of USG guidance was to know the angulation of the tandem required, the length of the tandem to be inserted, and the position of the uterus (acutely anteverted or retroverted). In another 12 patients, though the os could be identified visually, insertion of the uterine sound in the cervical canal was only possible with ultrasound guidance. This was because of the cervical canal stenosis due to radiation or disease induced fibrosis and greater extent of the residual disease. However, proper placement of the tandem applicator could only be performed with ultrasound guidance in these patients. In one of these 91 patients, although the cervical os was identifiable correctly with naked eye (which was again confirmed with USG), the uterine sound could not be passed even with ultrasound guidance, as the os was completely stenosed due to fibrosis. This patient was treated with interstitial brachytherapy. In another 4 patients, the cervix was completely flushed with vagina and the os was unidentifiable visually alone. In these patients, the cervical os was accurately identified and tandem was placed successfully by intraoperative ultrasound scanning. In another study patient, the cervical anatomy was completely destroyed by the tumor and the patient was treated with interstitial brachytherapy. Hence, a tedious insertion of the tandem with naked eye, a risk of uterine perforation, and inappropriate placement of the uterine tandem was avoided in 18 of the study patients (12 patients with stenosed os, where tandem insertion required USG guidance; 4 patients with cervix flushed with vagina, where USG guidance was necessary for os identification, and 2 patients, where intraoperative ultrasound was essential to understand the necessity for interstitial brachytherapy). Ultrasound guidance failed to identify the os in only one patient where the cervical anatomy was completely destroyed by the tumor.
In 15 study patients, the length of the uterine canal changed in the subsequent brachytherapy application. This was probably due to the shrinkage of the tumor near the external os. The variation in the tandem length inserted in these patients ranged from $0.25 \mathrm{~cm}$ to $0.75 \mathrm{~cm}$. Fortunately, none of the patients had uterine perforation in the study. The average applicator insertion time was approximately 27 minutes with routine real-time ultrasound guidance.

\section{Discussion}

Accurate placement of intracavitary applicators has been shown to be the most important prognostic factor in improving local tumor control in cervical cancer patients [8]. The reported risk of uterine perforation without ultrasound guidance ranges from $2-14 \%$ in various studies [7, $11,12,13,16]$. Uterine perforation causes patient discomfort and abdominal pain. It may also result in reapplication of the tandem applicator and cause treatment delay. Extended overall treatment time increases the chances of local failure [25]. Superior perforations though, can still be treated without loading dwell positions at the tip of the tandem. Alteration of the dwell time has been shown to significantly influence the dosimetric parameters in brachytherapy [26]. The benefit of ultrasound guided tandem placement in difficult cases have been previously demonstrated in many studies [9, 12, 16, 27, 28]. Nevertheless, very few studies have used routine ultrasound guidance for tandem placement in ICBT [29, 30]. Without ultrasound guided tandem placement, Granai et al. [16] reported $10 \%$ patients with uterine perforations. Kim et al. [13] reported uterine perforations in $2.3 \%$ of the 622 study patients. Even by placing metal clips on the uterine serosa to assess tandem placement, Matsuyama et al. [31] reported a $9.8 \%$ rate of uterine perforation without ultrasound 
guidance. While with the routine use of ultrasound guided tandem placement, Watkins et al. [29] and Schaner et al. [30], both reported only $1.4 \%$ rate of uterine perforation. Davidson et al., alike the current study, did not experience any uterine perforation at all in 21 patients (35 intracavitary insertions) with the use of ultrasound during tandem placement [19]. The routine use of real-time ultrasound guided ICBT was also found to shorten the time to complete the procedure. Quite similar to the current study, Davidson et al. have reported an average applicator insertion time of 26 minutes. The routine use of ultrasound guided tandem placement have also shown to reduce the dependency on gynecological oncologist [19]. Corn et al. [12] have demonstrated the use of ultrasound guided tandem placement in cases of cervical stenosis, undetermined orientation of the uterine canal, and in cases of previous perforation. Mayr et al. [27] demonstrated that ultrasound guidance facilitated accurate tandem placement in patients with retroverted uterus. The use of routine ultrasonography for tandem applicator placement is an accurate, cost-effective, easily accessible, and faster method of ICBT application [32] with lesser incidence of uterine perforations [33]. Thus, its use is even more applicable in the developing countries. The limitation of the study is that it does not compare USG guided tandem insertions directly with blind tandem insertions. Hence, the role of USG guided tandem insertion in preventing perforations cannot be commented on with statistical significance.

\section{Conclusions}

Accurate tandem insertion and its proper placement is of utmost importance in cervical ICBT. While blind tandem insertion on the basis of the feeling of slight resistance by the radiation oncologist have been used mostly throughout the world, the routine use of intraoperative real-time ultrasonography provides a better accuracy of tandem insertion, minimizing the risk of uterine perforation. Ultrasound guided tandem placement have also been found to be a faster method of ICBT application. It is cost-effective and readily available, even in most centers in the developing countries.

\section{Disclosure}

Authors report no conflict of interest.

\section{References}

1. Burden of HPV related cancers. Human Papillomavirus and Related Diseases Report India. Available from http://www. hpvcentre.net [last accessed on 15 October 2014].

2. Li Z, Yang S, Liu L, Han S. A comparison of concurrent chemoradiotherapy and radiotherapy in Chinese patients with locally advanced cervical carcinoma: a multi-center study. Radiat Oncol 2014; 9: 212.

3. Lukka H, Hirte H, Fyles A et al. Concurrent cisplatin-based chemotherapy plus radiotherapy for cervical cancer - a metaanalysis. Clin Oncol (R Coll Radiol) 2002; 14: 203-212.

4. Eifel PJ. Intracavitary brachytherapy in the treatment of gynecologic neoplasms. J Surg Oncol 1997; 66: 141-147.

5. Okuma K, Yamashita H, Kobayashi R et al. A study of highdose-rate intracavitary brachytherapy boost for curative treatment of uterine cervical cancer. J Contemp Brachytherapy 2015; 7: 128-134.

6. ICRU Report No. 38. Dose and volume specification for reporting Intracavitary therapy in Gynecology. International Commission on Radiation Units and Measurements, Bethesda 1985.

7. Barnes EA, Thomas G, Ackerman I et al. Prospective comparison of clinical and computed tomography assessment in detecting uterine perforation with intracavitary brachytherapy for carcinoma of the cervix. Int J Gynecol Cancer 2007; 17: 821-826.

8. Corn BW, Hanlon AL, Pajak TF et al. Technically accurate intracavitary insertions improve pelvic control and survival among patients with locally advanced carcinoma of the uterine cervix. Gynecol Oncol 1994; 53: 294-300.

9. Katz A, Eifel PJ. Quantification of intracavitary brachytherapy parameters and correlation with outcome in patients with carcinoma of the cervix. Int J Radiat Oncol Biol Phys 2000; 48: 1417-1425.

10. Cunningham DE, Stryker JA, Velkley DE et al. Routine clinical estimation of rectal, rectosigmoidal, and bladder doses from intracavitary brachytherapy in the treatment of carcinoma of the cervix. Int J Radiat Oncol Biol Phys 1981; 7: 653-660.

11. Jhingran A, Eifel PJ. Perioperative and postoperative complications of intracavitary radiation for FIGO stage I-III carcinoma of the cervix. Int J Radiat Oncol Biol Phys 2000; 46: 1177-1183.

12. Corn BW, Shaktman BD, Lanciano RM et al. Intra- and perioperative complications associated with tandem and colpostat application for cervix cancer. Gynecol Oncol 1997; 64: 224-229.

13. Kim RY, Levy DS, Brascho DJ et al. Uterine perforation during intracavitary application. Prognostic significance in carcinoma of the cervix. Radiology 1983; 147: 249-251.

14. Rotmensch J, Waggoner SE, Quiet C. Ultrasound guidance for placement of difficult intracavitary implants. Gynecol Oncol 1994; 54: 159-162.

15. Sahinler I, Cepni I, Oksuz DC et al. Tandem application with transvaginal ultrasound guidance. Int J Radiat Oncol Biol Phys 2004; 59: 190-196.

16. Granai CO, Doherty F, Allee P et al. Ultrasound for diagnosing and preventing malplacement of intrauterine tandems. Obstet Gynecol 1990; 75: 110-113.

17. Mahantshetty U, Khanna N, Swamidas J et al. Trans-abdominal ultrasound (US) and magnetic resonance imaging (MRI) correlation for conformal intracavitary brachytherapy in carcinoma of the uterine cervix. Radiother Oncol 2012; 102: 130-134.

18. Small W, Strauss JB, Hwang CS et al. Should uterine tandem applicators ever be placed without ultrasound guidance? No: a brief report and review of the literature. Int J Gynecol Cancer 2011; 21: 941-944.

19. Davidson M, Yuen J, D'Souza D et al. Optimization of highdose-rate cervix brachytherapy applicator placement: the benefits of intraoperative ultrasound guidance. Brachytherapy 2008; 7: 248-253.

20. Viswanathan AN, Erickson BA. Three-dimensional imaging in gynecologic brachytherapy: a survey of the American Brachytherapy Society. Int J Radiat Oncol Biol Phys 2010; 76: 104-109.

21. Tharavichitkul E, Wanwilairat S, Watcharawipha A et al. The effect of central shielding in the dose reporting for cervical cancer in EQD2 era. J Contemp Brachytherapy 2013; 5: 236-239.

22. ICRU Report \#38. Dose and volume specification for reporting intracavitary therapy in oncology. International Commission on Radiation Units and Measurements, Bethesda, 1985.

23. Viswanathan AN, Erickson BA. Three-dimensional imaging in gynecologic brachytherapy: a survey of the American 
Brachytherapy Society. Int J Radiat Oncol Biol Phys 2010; 76: 104-109.

24. Schindel J, Zhang W, Bhatia SK et al. Dosimetric impacts of applicator displacements and applicator reconstruction-uncertainties on 3D image-guided brachytherapy for cervical cancer. J Contemp Brachytherapy 2013; 5: 250-257.

25. Perez CA, Grigsby PW, Castro-Vita H et al. Carcinoma of the uterine cervix. I. Impact of prolongation of overall treatment time and timing of brachytherapy on outcome of radiation therapy. Int J Radiat Oncol Biol Phys 1995; 32: 1275-1288.

26. Ibhade OR, Oyeyemi OE, Idayat AB et al. Tandem-ring dwell time ratio in Nigeria: dose comparisons of two lading patterns in standard high-dose-rate brachytherapy planning for cervical cancer. J Contemp Brachytherapy 2015; 7: 161-170.

27. Mayr NA, Montebello JF, Sorosky JI et al. Brachytherapy management of the retroverted uterus using ultrasound-guided implant applicator placement. Brachytherapy 2005; 4: 24-29.

28. Irvin W, Rice L, Taylor P et al. Uterine perforation at the time of brachytherapy for carcinoma of the cervix. Gynecol Oncol 2003; 90: 113-122.

29. Watkins JM, Kearney PL, Opfermann KJ et al. Ultrasoundguided tandem placement for low-dose-rate brachytherapy in advanced cervical cancer minimizes risk of intraoperative uterine perforation. Ultrasound Obstet Gynecol 2011; 37: 241244.

30. Schaner PE, Caudell JJ, De Los Santos JF et al. Intraoperative ultrasound guidance during intracavitary brachytherapy applicator placement in cervical cancer: the University of Alabama at Birmingham experience. Int J Gynecol Cancer 2013; 23: 559-566.

31. Matsuyama T, Tsukamoto N, Matsukuma K et al. Uterine perforation at the time of brachytherapy for the carcinoma of the uterine cervix. Gynecol Oncol 1986; 23: 205-211.

32. Van Dyk S, Narayan K, Fisher R et al. Conformal brachytherapy planning for cervical cancer using transabdominal ultrasound. Int J Radiat Oncol Biol Phys 2009; 75: 64-70.

33. Van Dyk S, Bernshaw D. Ultrasound-based conformal planning for gynaecological brachytherapy. J Med Imaging Radiat Oncol 2008; 52: 77-84. 